\title{
ABSTRACT
}

\section{Descriptive Study on Reported Medication Error among Nurses in Queen Elizabeth II Hospital, Kota Kinabalu, Sabah}

Jenet Guan Chin*, Mary Tan, Stephanie Yvonnesky Francis, Siti Rahmah Idris, Mary Padtong, Kotulin Lotupas, Maglen Ginsos, Norizzati Cheng, Phyllis Bridget Philip

Nursing Unit, Hospital Queen Elizabeth II

*Corresponding author's email: jenetazmi@gmail.com

Keywords: medication error, safety practice, medication administration
Introduction: Medication error is a global issue. Despite, the various impacts on health and non-health, continuous monitoring, assessment and intervention are required to reduce the number of medication error. Precise information on the root cause of medication error in Hospital Queen Elizabeth II, Kota Kinabalu will aid in the preventative measures to reduce medication error among nurses. Thus, this study aims to describe the incident of medication errors among nurses. Methods: A retrospective cross-sectional study was conducted to review medication error incidents reports between 2015 to 2018. Data were analysed according to the type of error, day and shift of medication error occurred, causes and month of services. The collected data were analysed using descriptive statistics in SPSS 22. Results: A total of 54 reports was reviewed. The mean (SD) month of services among nurses involved in the medication error is 41.3 (24.9) months. The most common type of medication error is the wrong frequency with $23(42.6 \%)$ cases. Majority cases of medication error occurred in weekdays with 41 (75.9\%) cases and 24 (44.4\%) cases happen during the night shift. Poor communication among healthcare workers was the most commonly reported human error with 42 (77.8\%) reports, followed by $36(66.7 \%)$ reports of failure to comply standard of procedure in medication administration. Conclusion: Though this study found team factor is the recurrent causes, poorly designed work systems and 
Borneo Journal of Medical Sciences (BJMS), Special Issue, Volume 3, March 2020: 7 - 8

individual factor should be imperious as well. A qualitative study is required to understand more on nurse behaviour practice towards medication administration. The high authority plays an important role to monitor this matter to improve medication safety practice. 